\title{
Asociación entre migraña y enfermedad cardiovascular
}

\author{
Association between migraine and cardiovascular disease
}

Kurth T y col. BMJ 2016; 353:i2610.

\section{Objetivos}

Evaluar la asociación de la migraña con la enfermedad cardiovascular y la mortalidad cardiovascular en las mujeres.

\section{Diseño, lugar y pacientes}

Estudio prospectivo de cohortes del estudio Nurses' Health Study II. Se llevó a cabo en EE.UU. entre el año 1989 y 2011. Participa-ron 116.430 enfermeras mujeres de 25 a 42 años, de las cuales 889 fueron excluidas por presentar enfermedad cardiovascular o angina de pecho, quedando así 115.541. Al inicio las participantes completaron un cuestionario que incluía preguntas sobre antecedentes, estilo de vida y factores reproductivos, que fueron actualizando cada 2 años. En el cuestio-nario de 1989, 1993 y 1995 se les preguntó si un médico les había diagnosticado migraña en algún momento de sus vidas.

\section{Exposición}

A través del auto-reporte del diagnóstico de migraña, quedaron conformados dos grupos: uno con migraña y otro sin migraña. Se observó en cada uno de ellos las características de la población en cuanto a la prevalencia de factores de riesgo cardiovascular (FRCV) como hipertensión, tabaquismo, obesidad, uso de anticonceptivos orales, terapia de reemplazo hormonal, etc. y la incidencia de los resultados principales.

\section{Medición de resultados principales}

El resultado primario fue el desarrollo de enfermedad cardiovascular (ECV) mayor: una combinación de todos los eventos de infarto agudo de miocardio (IAM), accidente cerebrovascular (ACV) y muerte por estas causas. Como resultados secundarios se incluyeron IAM, ACV, angina de pecho, revascularización coronaria y mortalidad cardiovascular. Los datos fueron analizados a través de la evaluación de modificación de efecto que incluyó edad, colesterol elevado, diabetes, hipertensión, índice de masa corporal (IMC), tabaquismo, uso de terapia de reemplazo hormonal, anticonceptivos orales, aspirina, paracetamol, antiinflamatorios no esteroideos (AINE), entre otros.

\section{Resultados}

De las 115.541 mujeres que participaron del estudio, 1.731 $(15,2 \%)$ reportaron diagnóstico de migraña al inicio del estudio. Posteriormente 6.389 reportaron migraña durante el seguimiento y fueron incluidas en el grupo migraña para el análisis. Las mujeres con migraña presentaron mayor riesgo de desarrollar eventos cardiovasculares (ver Tabla 1).

Tabla 1: Probabilidad de desarrollar enfermedad cardiovascular en mujeres con migraña.

\begin{tabular}{c|c|c}
\hline $\begin{array}{c}\text { Condiciones asociadas } \\
\text { a migraña }\end{array}$ & HR (IC $\mathbf{9 5 \%}$ ) & $\mathbf{P}$ \\
\hline ECV mayor & $1,50(1,33$ a 1,69$)$ & $<0,01$ \\
\hline IAM & $1,39(1,18$ a 1,64$)$ & $<0,01$ \\
\hline ACV & $1,62(1,37$ a 1,92$)$ & $<0,01$ \\
\hline Angina/Revascularización & $1,73(1,29$ a 2.32$)$ & $<0,01$ \\
\hline Mortalidad cardiovascular & $1,37(1,02$ a 1,83$)$ & 0,04 \\
\hline
\end{tabular}

HR: Hazard Ratio ajustado por edad, colesterol elevado, diabetes, hipertensión arterial, índice de masa corporal, estatus tabáquico, consumo de alcohol, actividad física, uso de terapia hormonal de reemplazo, menopausia, uso previo de anticonceptivos orales, uso de aspirina, paracetamol o anti-inflamatorios no esteroideos, e historia familiar de infarto de miocardio antes de los 60 años de edad. ECV: enfermedad cardiovascular. IAM: infarto aguso de miocardio. ACV: accidente cerebro vascular.

\section{Conclusión}

Las mujeres que tienen migraña presentan un perfil de factores de riesgo cardiovasculares más desfavorable que aquellas que no tienen migraña. Presentan un $50 \%$ más de riesgo de enfermedad cardiovascular mayor, siendo el ACV el que más se asocia.

Conflicto de intereses/Fuente de financiamiento: los autores no recibieron financiamiento específico para realizar este estudio.

\section{Comentario}

La migraña, principalmente aquella con aura, ha sido históricamente asociada con el riesgo de $\mathrm{ACV}^{1,2}$. En este estudio no se discriminó entre migraña con y sin aura. Tampoco hay información acerca de la frecuencia de los síntomas, de la intensidad del dolor o del uso de tratamiento preventivo. Otro comentario a destacar es que tanto los FRCV como el diagnóstico de migraña fueron por auto-reporte y no fueron constatados en forma objetiva a través de registros en historia clínica u otras fuentes. Si bien este trabajo documenta la asociación que existe entre la migraña y la enfermedad cardiovascular, quedan aún pendientes ciertos interrogantes: el tratamiento de esta entidad, ¿disminuiría el riesgo de enfermedad cardiovascular? ¿Qué mensaje le damos a nuestras pacientes con migraña?

\section{Conclusiones del comentador}

Dado que se trata de un gran estudio prospectivo de cohorte, con una población relativamente cautiva interrogada bienalmente y con un exhaustivo ajuste, consiste en evidencia suficiente para avalar la asociación independiente entre migraña y riesgo cardiovasculares. Si bien no hay evidencia específica de reducción del riesgo cardiovascular con el tratamiento de la migraña, éste mejora la calidad de vida de las personas que la padecen.Se debería recomendar un estilo de vida saludable para mejorar el perfil de riesgo cardiovascular a los pacientes con migraña o sin ella.

Camila Volij [ Servicio de Medicina Familiar y Comunitaria del Hospital Italiano de Buenos Aires camila.volij@ hospitalitaliano.org.ar ]

Volij C. Asociación entre migraña y enfermedad cardiovascular. Evid Actual Pract Ambul 2018;21(3):85. Comentado de: Kurth T y col. Migraine and risk of cardiovascular disease in women: prospective cohort study. BMJ 2016;353:i2610. PMID: 27247281. 